'Unidad de Estudios

Psicosociales, Facultad de Psicología, Universidad Nacional Autónoma de México, Ciudad de México, México.

2Departamento de Psicología, Facultad de Educación, Ciencias

Sociales y Humanidades,

Universidad de La Frontera, Temuco, Chile.

${ }^{3}$ Facultad de Ciencias Sociales, Carrera de Psicología, Universidad Católica de Temuco, Chile. ${ }^{4}$ Culture and Behavior Laboratory, Loma Linda University, USA. ${ }^{5}$ Laboratorio de Estrés y Salud. Doctorado en Psicología, Facultad de Educación, Ciencias Sociales y Humanidades, Universidad de La Frontera, Temuco, Chile. apsicólogo ${ }^{\mathrm{b}} \mathrm{PhD}$

Recibido el 11 de julio de 2017, aceptado el 5 de marzo de 2018.

Correspondencia a: Dra. María José Baeza Rivera Carrera de Psicología Universidad Católica de Temuco. Manuel Montt 56. Temuco, Chile. Teléfono de contacto: $+56452685079$ maria.baeza@uct.cl

\section{Identificación y medición de creencias culturales sobre los médicos: construcción y validación de un instrumento}

\author{
MARÍA JOSÉ BAEZA-RIVERA ${ }^{1,2,3 a, b}$, HÉCTOR BETANCOURT $^{2, a, b}$, \\ NATALIA SALINAS-OÑATE ${ }^{2, \mathrm{a}, \mathrm{b}}$, MANUEL S. ORTIZ $Z^{2,5, \mathrm{a}, \mathrm{b}}$, \\ OLIVIA MARÍN ${ }^{2, a}$, GISSEL VALLE ${ }^{2, a}$
}

\section{Development of an instrument to assess cultural beliefs about physicians}

Background: Beliefs about professionals' healthcare may influence healthcare behaviors. Such beliefs are in part the result of the interactions that professionals have with their patients. Recent studies highlight the importance of beliefs about physicians, their effect on health-care behaviors, and the requirement of culturally appropriate tools to measure such beliefs. Aim: To develop and validate a culturally appropriate instrument to measure beliefs about physicians. Material and Methods: Based on a "bottom-up" methodology, a culturally pertinent scale of beliefs about physicians was developed and then validated by expert judges. The resulting scale, with 26 items, was applied to 337 participants aged $31 \pm 7$ years (85\% women). Results: Two factors, grouping 24 items, emerged from the exploratory factor analysis. The first was called negative beliefs about doctors (Cronbach's $\alpha=0.96$ ) and the second was called positive beliefs about doctors (Cronbach's $\alpha=0.95$ ). Both factors explain $70 \%$ of the scale variance. Conclusions: The devised instrument has adequate psychometric properties and is also culturally relevant. It allows the assessment of cultural beliefs about physicians.

(Rev Med Chile 2018; 146: 308-314)

Key words: Culture; Physician-Patient Relations; Psychometrics; Weights and Measures.
$\mathrm{L}$ as creencias que tienen los pacientes respecto a los profesionales de la salud influyen en la percepción de la atención y en la relación que se establece con ellos, además determinan conductas de prevención y adhesión a los tratamientos ${ }^{1,2}$. La definición clásica de creencias planteada por Moscovici (1979), indica que éstas son representaciones sociales, explicaciones, conceptos o enunciados socialmente compartidos por un grupo cultural. Desde el ámbito de la salud, son relevantes por su relación con las conductas de las personas en este contexto, las que son influidas por las experiencias que se ha tenido en la interacción con los profesionales y el sistema de salud ${ }^{4}$.
Estudios en mujeres Latinas y Anglo sobre conductas de screening para cáncer de mama, dan cuenta de que las creencias culturales positivas sobre los profesionales de salud influyen directamente en la continuidad de los exámenes preventivos $^{4}$. Por el contrario, el ser víctima de malos tratos (ej. largos tiempos de espera, faltas de respeto), generan creencias y expectativas negativas, lo que lleva a una menor probabilidad de continuar las atenciones de salud, determinando el curso de su auto cuidado ${ }^{1,5,6}$.

Respecto de las creencias sobre los médicos, investigaciones realizadas en Estados Unidos de Norteamérica con mujeres latinas y de nivel 
socioeconómico bajo señalan, que los profesionales son "severos" y "fríos"7, "poco confiables", faltos de preocupación y compasión". En Chile las pacientes califican la relación con su médico como impersonal, fría y poco centrada en lo que realmente necesitan ${ }^{10}$.

En los países más desarrollados ha aumentado el interés por considerar el punto de vista de los pacientes, contribuyendo a mejorar los procesos de gestión sanitaria. Sin embargo, en Chile es un desafío medir las creencias que los pacientes tienen sobre los profesionales de la salud, por lo que habitualmente se evalúa la percepción de la calidad de la atención y satisfacción de los usuarios, desconociendo posibles causales (Ej., creencias asociadas a los profesionales, a las experiencias y sus efectos psicológicos).

Actualmente, existe poca evidencia respecto de instrumentos locales que permitan dar cuenta de las opiniones o creencias que poseen los pacientes sobre los profesionales que atienden en los servicios de salud, por lo tanto, contar con medidas que posean adecuadas propiedades psicométricas y que además sean culturalmente pertinentes se hace una necesidad.

Considerando los antecedentes expuestos, el objetivo de esta investigación es construir y validar un instrumento culturalmente pertinente que mida las creencias sobre los médicos. Para ello se utiliza un enfoque metodológico "bottom-up" descrito por Betancourt et al. (2010).

El estudio se dividió en tres etapas, la primera tuvo por objetivo identificar creencias culturales sobre los médicos, la segunda, construir y pilotear un instrumento y en la etapa final estimar las propiedades psicométricas de la escala de creencias sobre los médicos. Específicamente, se investigan las creencias que tienen los pacientes que asisten a los controles de niño sano, una actividad que representa el primer acercamiento de la población hacia el sistema de salud ${ }^{10}$, dirigida a promover la salud integral de los niños y detectar anomalías ${ }^{11}$ para derivar oportunamente ${ }^{12}$.

\section{Primera fase: Identificación de creencias culturales}

\section{Materiales y Método}

Participantes: Por medio de un muestreo de casos por criterio se entrevistó a 32 personas de la Región de La Araucanía en base a las siguientes categorías poblacionales: a) sistema de salud (público y privado); b) etnicidad (mapuche y no mapuche); y c) edad (categorizada en dos niveles: de 15 a 30 años, y 30 años o más) ya que éstas son fuentes de variación cultural, procurando tener la mayor cantidad de personas por categorías.

Instrumentos: Se utilizó un formato de entrevista semiestructurada con preguntas abiertas sobre creencias socialmente compartidas acerca de los médicos (ej. “QQué es lo primero que viene a su mente cuando piensa en los médicos?"), basado en el protocolo implementado por Betancourt y colaboradores ${ }^{9}$.

Procesamiento y análisis de respuestas: Las entrevistas fueron grabadas, luego fueron transcritas y la información obtenida se trianguló para evitar sesgos y cumplir con el criterio de rigor de comprobabilidad ${ }^{13}$. Posteriormente, se sometió a análisis de contenido simple, es decir, se extrajeron los principales contenidos y con aquellas categorías que tenían mayor frecuencia se desarrollaron ítems de respuesta cerrada y tipo Likert para la segunda etapa del estudio.

\section{Resultados}

Como se aprecia en la Figura 1, las creencias socialmente compartidas sobre los médicos, se centraron principalmente en las características personales y el trato que entregan a sus pacientes. Se identifican creencias asociadas a características personales de los médicos, las que pueden ser positivas (ej. son preocupados y se interesan por sus pacientes), como negativas, (ej. se creen superiores). En relación al último tópico, se mencionan diferencias existentes entre los médicos antiguos y nuevos y diferencias por sexo.

\section{Segunda fase: Desarrollo y pilotaje del instrumento}

\section{Desarrollo de ítems y validación de contenido}

En base a los contenidos obtenidos de la primera fase, se crearon 23 reactivos para medir las creencias sobre los médicos, con un formato de respuesta tipo Likert de cinco puntos $(1=$ Totalmente en desacuerdo a $5=$ Totalmente de acuerdo).

La versión preliminar de la escala fue sometida a validación de contenido, por nueve jueces expertos, quienes de manera independiente evaluaron 


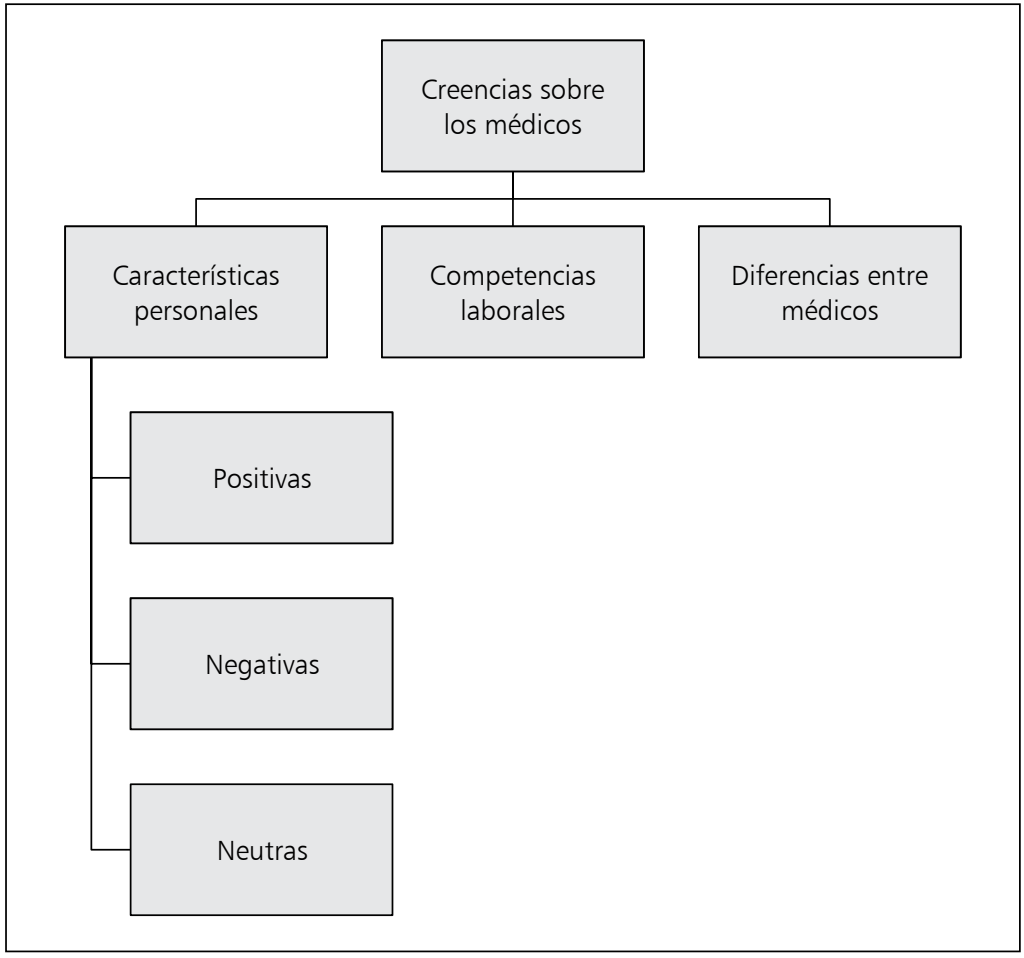

Figura 1. Creencias sobre médicos.

la coherencia, suficiencia y claridad de cada uno de los reactivos. El grado de concordancia de las puntuaciones dadas por cada juez fueron estimadas con el coeficiente V de Aiken ${ }^{14,15}$, seleccionándose únicamente ítems cuyo valor fue mayor o igual a 0,80 .

\section{Pilotaje de instrumentos}

Con el fin de probar el comportamiento del instrumento en población similar a la que se utilizaría, la escala resultante fue sometida a un pilotaje con 105 cuidadores de niños de entre 1 mes y 12 años. Como criterio de inclusión se consideró que hubiesen tenido al menos una experiencia en los sistemas de salud. Luego de los análisis y en base a los antecedentes teóricos y empíricos, se realizaron nuevas modificaciones a la escala, quedando finalmente compuesta por 26 reactivos.

\section{Tercera fase: Evaluación de las propiedades psicométricas de la escala}

Materiales y Método

Participantes. Por medio de un muestreo intencionado, se contactó a 337 madres, padres o cuidadores de niños entre 1 mes y 7 años. Como criterio de inclusión se consideró que hubiesen tenido al menos una experiencia en los sistemas de salud con médico para la atención de su hijo/a. El promedio de edad de los participantes fue 31 años (DS $=7,46)$ y de los hijos/as fue de 25 meses $(\mathrm{DS}=17,01)$. La mayoría de quienes contestaron fueron mujeres $(85 \%), 57 \%$ reportó ser usuario del sistema público de salud y $32 \%$ declaró pertenecer a la etnia mapuche.

Instrumentos. Datos demográficos de los participantes, entre ellos la edad, sexo, procedencia, sistema de salud en el que se atiende, nivel educacional, ingreso, nivel socioeconómico de acuerdo a escala ESOMAR ${ }^{16}$ y etnia.

Escala de creencias sobre los médicos, finalmente constituida por 26 ítems donde los participantes deben indicar su grado de acuerdo con respecto a las creencias sobre los médicos por medio de un formato de respuesta tipo Likert, de 5 puntos ( 1 = muy en desacuerdo a $5=$ muy de acuerdo $)$, donde altos puntajes indican mayor presencia de las creencias (ej. "Los médicos sólo se interesan en ganar dinero"). 


\section{Procedimiento de recolección de datos}

Los participantes fueron contactados en los Centros de Salud Familiar (CESFAM) y jardines infantiles de la comuna de Temuco, previa autorización del Departamento de Salud Municipal de Temuco y de la Junta Nacional de Jardines Infantiles (JUNJI). Los participantes firmaron el consentimiento aprobado por el Comité de Ética del Servicio de Salud Araucanía Sur y posteriormente contestaron el instrumento. Finalmente firmaron un recibo simple por la retribución económica que se les entregó por el tiempo dedicado a la investigación.

\section{Análisis de datos}

Los datos se analizaron en el programa estadístico SPSS 20.0. En primer lugar, se realizaron análisis descriptivos para caracterizar la muestra. Posteriormente, se llevó a cabo el análisis factorial exploratorio, para evaluar la estructura factorial. Para ello se evaluó la capacidad de factorizar de la matriz por medio de las medidas de adecuación muestral de Kaiser Meyer-Olkin (KMO) y la prueba de Bartlett, esperando resultados ser mayores a 0,80 para $\mathrm{KMO}^{17}$, y rechazar la hipótesis nula respecto de que la matriz es de identidad para Bartlet $^{18}$. Posteriormente, utilizando el método máxima verosimilitud y rotación oblimin se buscó la mejor solución, considerando criterios estadísticos y teóricos, tales como: a) seleccionar sólo los factores que tengan autovalores mayores a uno; b) eliminar los ítems cuya carga factorial era menos a 0,$30 ; \mathrm{yc}$ ) eliminar los factores compuestos por menos de tres ítems. Una vez identificados los factores, se les designó un nombre. Luego para evaluar la confiabilidad de las escalas, se realizaron los análisis de consistencia interna por medio del alpha de Cronbach ${ }^{19}$.

\section{Resultados}

Los resultados dan cuenta de una matriz factorizable, específicamente, la prueba KMO fue de 0,85 y la prueba de esfericidad de Bartlett fue significativa $\left(\chi^{2}=1797,647 ; \mathrm{p}<0,001\right)$. La estructura factorial da cuenta de dos factores que explican $70,31 \%$ de la varianza, el primero de ellos se denominó "Creencias negativas sobre los médicos", compuesto por 13 reactivos, explicando $56,6 \%$ de la varianza con una consistencia interna de 0,96 . El segundo factor se denominó "Creencias positivas sobre los médicos", compuesto por 11 ítems, explicando $13,7 \%$ de la varianza y con una confiabilidad de 0,95 . Se eliminaron dos ítems por criterios teóricos (más detalles en Tabla 1).

Además se pueden apreciar en la Tabla 2 las medias y desviaciones típicas de los ítems de la escala, donde el reactivo que obtiene el promedio más alto es "los médicos atienden de buena forma a sus pacientes" $(\mathrm{M}=3,68, \mathrm{DT}=0,88)$ y el promedio más bajo es "los médicos tratan mal a sus pacientes” $(\mathrm{M}=2,26, \mathrm{DT}=0,84)$.

\section{Tabla 1. Estructura factorial, cargas factoriales, varianza explicada y confiabilidad de la Escala de} Creencias sobre Médicos

\begin{tabular}{|c|c|c|c|}
\hline$n$ & Ítem & $\begin{array}{c}\text { Factor creencias } \\
\text { negativas }\end{array}$ & $\begin{array}{c}\text { Factor creencias } \\
\text { positivas }\end{array}$ \\
\hline 17 & Son enojones con sus pacientes & 0,94 & \\
\hline 18 & Son presumidos con sus pacientes & 0,94 & \\
\hline 19 & Son mañosos con sus pacientes & 0,93 & \\
\hline 20 & Tienen poca paciencia con sus pacientes & 0,92 & \\
\hline 16 & Son bruscos con sus pacientes & 0,90 & \\
\hline 5 & Discriminan a sus pacientes & 0,86 & \\
\hline 15 & Tratan mal a sus pacientes & 0,85 & \\
\hline 12 & Sólo se interesan en ganar dinero & 0,84 & \\
\hline 2 & Retan a sus pacientes cuando no cumplen con las indicaciones & 0,82 & \\
\hline 6 & Tienen mala voluntad para explicar o contestar preguntas a sus pacientes & 0,76 & \\
\hline 13 & Se creen superiores a los demás & 0,75 & \\
\hline 14 & Hacen diagnósticos erróneos & 0,72 & \\
\hline 1 & Se vuelven insensibles al dolor ajeno con el paso del tiempo & 0,63 & \\
\hline
\end{tabular}


Tabla 1. (Continuación)

\begin{tabular}{|c|c|c|c|}
\hline $\mathbf{n}$ & ítem & $\begin{array}{c}\text { Factor creencias } \\
\text { negativas }\end{array}$ & $\begin{array}{c}\text { Factor creencias } \\
\text { positivas }\end{array}$ \\
\hline 21 & Están disponibles para sus pacientes & & 0,71 \\
\hline 10 & Tienen un trato cercano con sus pacientes & & 0,64 \\
\hline 8 & Se interesan o preocupan por sus pacientes & & 0,63 \\
\hline 7 & Tienen vocación para atender a sus pacientes & & 0,56 \\
\hline 9 & Dan confianza a sus pacientes & & 0,55 \\
\hline 25 & Son cercanos con sus pacientes & & 0,52 \\
\hline 11 & Son amables con sus pacientes & & 0,51 \\
\hline 23 & Son acogedores o cariñosos con sus pacientes & & 0,45 \\
\hline 24 & Son comprometidos con sus pacientes & & 0,45 \\
\hline 26 & Son capaces de entender a sus pacientes & & 0,38 \\
\hline \multirow[t]{3}{*}{22} & Atienden de buena forma a sus pacientes & & 0,31 \\
\hline & Varianza Explicada & $56,6 \%$ & $13,7 \%$ \\
\hline & $\alpha$ de Cronbach & 0,961 & 0,951 \\
\hline
\end{tabular}

Fuente: Elaboración propia.

Tabla 2. Promedios y desviaciones típicas de la Escala de Creencias sobre Médicos

\begin{tabular}{|c|c|c|c|}
\hline $\mathbf{n}$ & Ítem & Media & Desviación típica \\
\hline 1 & Se vuelven insensibles al dolor ajeno con el paso del tiempo & 3,33 & 1,25 \\
\hline 2 & Retan a sus pacientes cuando no cumplen con las indicaciones & 3,06 & 1,19 \\
\hline 5 & Discriminan a sus pacientes & 2,39 & 1,01 \\
\hline 6 & Tienen mala voluntad para explicar o contestar preguntas a sus pacientes & 2,58 & 1,17 \\
\hline 7 & Tienen vocación para atender a sus pacientes & 3,54 & 1,00 \\
\hline 8 & Se interesan o preocupan por sus pacientes & 3,51 & 0,96 \\
\hline 9 & Dan confianza a sus pacientes & 3,66 & 0,94 \\
\hline 10 & Tienen un trato cercano con sus pacientes & 3,31 & 1,00 \\
\hline 11 & Son amables con sus pacientes & 3,60 & 0,89 \\
\hline 12 & Sólo se interesan en ganar dinero & 3,02 & 1,03 \\
\hline 13 & Se creen superiores a los demás & 3,03 & 1,15 \\
\hline 14 & Hacen diagnósticos erróneos & 2,76 & 1,07 \\
\hline 15 & Tratan mal a sus pacientes & 2,26 & 0,84 \\
\hline 16 & Son bruscos con sus pacientes & 2,33 & 1,00 \\
\hline 17 & Son enojones con sus pacientes & 2,40 & 1,01 \\
\hline 18 & Son presumidos con sus pacientes & 2,36 & 0,98 \\
\hline 19 & Son mañosos con sus pacientes & 2,44 & 1,02 \\
\hline 20 & Tienen poca paciencia con sus pacientes & 2,61 & 1,09 \\
\hline 21 & Están disponibles para sus pacientes & 3,29 & 1,08 \\
\hline 22 & Atienden de buena forma a sus pacientes & 3,68 & 0,88 \\
\hline 23 & Son acogedores o cariñosos con sus pacientes & 3,30 & 0,98 \\
\hline 24 & Son comprometidos con sus pacientes & 3,40 & 0,94 \\
\hline 25 & Son cercanos con sus pacientes & 3,23 & 0,98 \\
\hline 26 & Son capaces de entender a sus pacientes & 3,41 & 1,00 \\
\hline
\end{tabular}

Fuente: Elaboración propia. 


\section{Discusión}

A través de este estudio se creó y validó una escala culturalmente pertinente para medir creencias sobre los médicos, compuesta por dos factores, el primer factor representa creencias negativas sobre los médicos y el segundo creencias positivas, ambos factores tienen un adecuado número de ítems, excelentes índices de confiabilidad y explican un alto porcentaje de varianza (70\%), dando cuenta de adecuadas propiedades psicométricas (confiabilidad y validez estructural). Además es de fácil administración y puntuación, lo cual hacen de esta escala un instrumento propicio para ser utilizado en investigaciones donde se incorporen variables culturales en el ámbito de la salud.

Respecto del proceso de creación y validación del instrumento, éste fue riguroso pasando por diversas etapas, donde se identificaron las creencias culturalmente pertinentes sobre los médicos. Lo anterior garantiza la equivalencia de la medición, es decir se evalúe realmente los significados que tienen los conceptos para las personas ${ }^{9}$ (pertinencia cultural). Además, el proceso de jueces expertos y pilotaje permitieron desechar aquellos ítems que no resultaron adecuados y perfeccionar los reactivos en cuanto a redacción y lenguaje, acumulando evidencia empírica para disminuir los sesgos del instrumento.

Es importante mencionar que el tamaño muestral para las diversas etapas del proceso fue el adecuado, puesto que en la primera fase se procuró saturar contenido, posteriormente en la tercera fase se utilizó como criterio que fuesen al menos diez personas por cada ítem de la escala.

En relación a los promedios, llama la atención que para las personas lo más relevante sea que les atiendan de buena manera, mientras que lo que presenta menor promedio sea que los médicos les discriminen, ambos elementos asociados al trato recibido.

Otra contribución es la identificación de creencias culturales, las positivas pueden ser reforzadas, favoreciendo que los pacientes puedan tener mejores resultados en salud, por su asociación comportamientos de cuidado de la salud. Más importante aún es poder identificar creencias negativas, ya que indirectamente podría dar cuenta de cómo los médicos están ejerciendo su labor, pues estas creencias derivan en buena medida de la evaluación que el paciente hace del desempeño del médico. Lo anterior es importante, considerando que existe cierta reticencia por parte de los médicos para recibir retroalimentación e incorporar cambios en sus atenciones médicas ${ }^{20}$.

Las creencias se perpetúan al traspasarse de una persona a otra (socialización), llegando incluso a formar estereotipos, los que finalmente pueden predisponer la búsqueda de atenciones de salud. Es sabido que las creencias que los pacientes tienen sobre los profesionales de la salud son predictores de la relación médico-paciente y de las conductas de cuidado y prevención, por lo tanto, considerar estas variables en estudios permite contribuir a comprender las interacciones entre profesionales y pacientes, pudiendo de esta manera mejorar la calidad de los encuentros médicos y favorecer las conductas de cuidado de los usuarios.

Una de las limitaciones fue que la mayoría de las personas que participaron fueron mujeres, de esta manera futuras líneas de investigación podrían centrarse en estudiar el comportamiento psicométrico de la escala con población de diferentes características.

Este tipo de investigaciones dan cuenta de la importancia de contar con instrumentos culturalmente pertinentes para ser utilizados en estudios donde se consideren variables culturales en contextos de salud.

Agradecimientos: Este estudio fue parte de la tesis doctoral de la Dra. María José Baeza-Rivera, desarrollada en la Universidad de La Frontera y recibió financiamiento por parte de la Comisión Nacional de Investigación Científica y Tecnológica, número de folio 21140104 .

\section{Referencias}

1. Betancourt H, Flynn P, Ormseth S. Healthcare Mistreatment and Continuity of Cancer Screening Among Latino and Anglo American Women in Southern California. Women Health 2011; 51 (1): 1-24.

2. Fassaert T, Hesselink AE, Verhoeff AP. Acculturation and use of health care services by Turkish and Moroccan migrants: a cross-sectional population-based study. BMC Public Health 2009; 9 (1): 1.

3. Moscovici S. El psicoanálisis, su imagen y su público. Huemul S.A. Buenos Aires, Argentina; 1979.

4. Amador JA, Flynn PM, Betancourt H. Cultural beliefs about health professionals and perceived empathy 
influence continuity of cancer screening following a negative encounter. J Behav Med 2015; 38 (5): 798-808.

5. Abraído-Lanza AF, Céspedes A, Daya S, Flórez KR, White K. Satisfaction with health care among Latinase. J Health Care Poor Underserved 2011; 22 (2): 491-505.

6. Lynn P, Noor S, Brandt J. Somali immigrant women and the American health care system: discordant beliefs, divergent expectations, and silent worries. Soc Sci Med 2010; 71 (2): 353-61.

7. Moy B, Park ER, Feibelmann S, Chiang S, Weissman JS. Barriers to repeat mammography: cultural perspectives of African-American, Asian, and Hispanic women. Psycho-Oncology 2006; 15 (7): 623-34.

8. Buki LP, Borrayo EA, Feigal BM, Carrillo IY. Are all latinas the same? Perceived breast cancer screening barriers and facilitative conditions. Psychol Women Q 2004; 28 (4): 400-11.

9. Betancourt H, Flynn P, Riggs M, Garberoglio C. A cultural research approach to instrument development: the case of breast and cervical cáncer screening among Latino and Anglo women. Heal Educ Res 2010; 25 (6): 991-1007.

10. Alarcón AM, Vidal AC. Dimensiones culturales en el proceso de atención primaria infantil: perspectivas de las madres. Salud Publica Mex 2005; 47 (6): 440-6.

11. Ministerio de Salud (MINSAL). Norma técnica para la supervisión de niños y niñas de 0 a 9 años en la atención primaria de salud. 2013. Disponible en: www.crececontigo.gob.cl.

12. Szot J. Reseña de la salud pública materno-infantil chilena durante los últimos 40 años: 1960-2000. Rev Chil Obstet Ginecol 2002; 67 (2): 129-35.

13. Guba EG. Criterios de credibilidad en la investigación naturalista. In: Sacristán JG, Gómez AP, editors. La enseñanza: su teoría y su práctica. Akal. Madrid; 1989. p. 148-65.

14. Aiken L. Three Coeficients for Analyzing the Reliability and Validity of Ralings. Educ Psychol Meas 1985; 45: 131-42.

15. Aiken L. Content Validity and Reliability of Single Items or Questionnaire. Educ Psychol Meas 1980; 40: 955-9.

16. Adimark. El Nivel Socio Económico ESOMAR, Manual de Aplicación. 2000. Disponible en www.adimark.cl.

17. Kaiser HF. A second generation Little jiffy. Psychometrika 1970; 35 (4): 401-15.

18. Bartlett MS. Tests of significance in factor analysis. Br J Stat Psychol 1950; 3 (2): 77-85.

19. Cronbach LJ. Coefficient alpha and the internal structure of tests. Psychometrika 1951; 16: 297-334.

20. Millenson ML. Demanding medical excellence: Doctors and accountability in the information age. University of Chicago Press, editor. Chicago; 1997. 